\title{
Optical fibers for endoscopic high-power Er:YAG laserosteotomy
}

\author{
Lina M. Beltrán Bernal $\odot$, a,* Ferda Canbaz $\odot$, a Salim E. Darwiche $\odot, \stackrel{\text { b,c }}{ }$

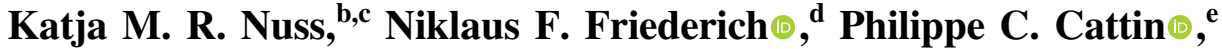 \\ and Azhar Zam ${ }^{\mathbf{a}}$,* \\ ${ }^{a}$ University of Basel, Department of Biomedical Engineering, Faculty of Medicine, \\ Biomedical Laser and Optics Group, Allschwil, Switzerland

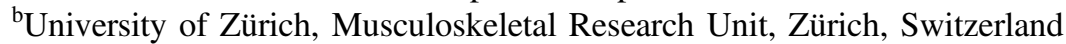 \\ ${ }^{c}$ University of Zürich, Center for Applied Biotechnology and Molecular Medicine, \\ Zürich, Switzerland \\ ${ }^{\mathrm{d}}$ University of Basel, Department of Biomedical Engineering, Faculty of Medicine, \\ Center of Biomechanics and Biocalorimetry, Allschwil, Switzerland \\ ${ }^{\mathrm{e}}$ University of Basel, Department of Biomedical Engineering, Faculty of Medicine, \\ Center for medical Image Analysis and Navigation, Allschwil, Switzerland
}

\begin{abstract}
Significance: The highest absorption peaks of the main components of bone are in the midinfrared region, making Er:YAG and $\mathrm{CO}_{2}$ lasers the most efficient lasers for cutting bone. Yet, studies of deep bone ablation in minimally invasive settings are very limited, as finding suitable materials for coupling high-power laser light with low attenuation beyond $2 \mu \mathrm{m}$ is not trivial.

Aim: The first aim of this study was to compare the performance of different optical fibers in terms of transmitting Er:YAG laser light with a $2.94-\mu \mathrm{m}$ wavelength at high pulse energy close to $1 \mathrm{~J}$. The second aim was to achieve deep bone ablation using the best-performing fiber, as determined by our experiments.

Approach: In our study, various optical fibers with low attenuation $(\lambda=2.94 \mu \mathrm{m})$ were used to couple the Er:YAG laser. The fibers were made of germanium oxide, sapphire, zirconium fluoride, and hollow-core silica, respectively. We compared the fibers in terms of transmission efficiency, resistance to high Er:YAG laser energy, and bending flexibility. The best-performing fiber was used to achieve deep bone ablation in a minimally invasive setting. To do this, we adapted the optimal settings for free-space deep bone ablation with an Er:YAG laser found in a previous study.
\end{abstract}

Results: Three of the fibers endured energy per pulse as high as $820 \mathrm{~mJ}$ at a repetition rate of $10 \mathrm{~Hz}$. The best-performing fiber, made of germanium oxide, provided higher transmission efficiency and greater bending flexibility than the other fibers. With an output energy of $370 \mathrm{~mJ}$ per pulse at $10 \mathrm{~Hz}$ repetition rate, we reached a cutting depth of $6.82 \pm 0.99 \mathrm{~mm}$ in sheep bone. Histology image analysis was performed on the bone tissue adjacent to the laser ablation crater; the images did not show any structural damage.

Conclusions: The findings suggest that our prototype could be used in future generations of endoscopic devices for minimally invasive laserosteotomy.

(C) The Authors. Published by SPIE under a Creative Commons Attribution 4.0 Unported License. Distribution or reproduction of this work in whole or in part requires full attribution of the original publication, including its DOI. [DOI: 10.1117/1.JBO.26.9.095002]

Keywords: laser ablation of bone; Er:YAG laser; optical fiber; germanium oxide fiber; sapphire fiber; zirconium fluoride fiber; hollow-core silica waveguide; deep bone ablation; minimally invasive laserosteotomy.

Paper 210145R received May 1, 2021; accepted for publication Aug. 16, 2021; published online Sep. 13, 2021.

*Address all correspondence to Lina M. Beltrán Bernal, lina.beltran@unibas.ch; Azhar Zam, azhar.zam@unibas.ch 


\section{Introduction}

In the field of osteotomy, the use of lasers has been studied for several years ${ }^{1-8}$ however, some early clinical studies showed severe collateral damage and a prolonged healing process. ${ }^{9}$ In response, efforts have been made to optimize laser systems intended for laserosteotomy. For example, modern Er:YAG laser systems have been used to remove intraoral hard tissue in humans, without showing damage in subsequent histological analyses. ${ }^{10}$ More recent studies have shown how the use of new irrigation and temperature feedback detection systems for Er:YAG laserosteotomy can help achieve safe, deep bone ablation. ${ }^{11}$ Other results from a robotic free-space laser device, CARLO ${ }^{\circledR}$, based on an Er:YAG laser, show potential for use in osteotomy applications. The CARLO laser device was used for the first clinical bone surgery worldwide, where the functionality of the patient's jaw was improved. After years of investigation in the field, the device was used in July 2019 for an in vivo mid-face osteotomy at the Department of Oral Maxillofacial Surgery, University Hospital Basel, Switzerland. 7,12,13

Several aspects make lasers attractive for tissue ablation. It is a safer procedure with respect to preventing bacterial and viral infection during surgery because of the contactless nature of the laser-tissue interaction. Lasers also make it easier to achieve the flexible cuts (various shapes and curves) desired during surgery, especially for cutting hard tissue. Although surgery with conventional mechanical tools has evolved to be as minimally invasive as possible, the flexibility provided by these tools remains limited. ${ }^{14}$ Endoscopic devices have emerged for use in minimally invasive procedures. These devices provide solutions for diagnosis and microsurgeries inside the body where other tools cannot reach. ${ }^{15,16}$ For endoscopic laser surgery involving hard tissues, some studies have shown the benefits of using fiber-coupled or fiber-based lasers. ${ }^{17,18}$ Lasers working from $600 \mathrm{~nm}$ up to $2 \mu \mathrm{m}$ can be easily coupled in low-OH silica fibers due to their low attenuation in this wavelength region. For instance, a Ho:YAG laser at a wavelength of $2.12 \mu \mathrm{m}$ has been widely used for lithotripsy, ${ }^{19}$ it can be used in endoscopic applications by means of silica fibers, as can the thulium laser at a wavelength of $1.908 \mu \mathrm{m} .{ }^{20}$ For endoscopic surgical applications requiring deeper hard-tissue ablation, such as a typical maxillofacial surgery on the jaw or during knee surgery, a laser delivering more energy per pulse through the optical fiber is needed. We return to the Er:YAG laser, which has shown outstanding results for deep bone ablation. ${ }^{10,11}$ Throughout the history of fiber research, finding materials for coupling laser light with low attenuation around $3 \mu \mathrm{m}$ has not been trivial. Today, however, some materials, such as sapphire, fluoride, ${ }^{18}$ and germanium oxide fibers, ${ }^{17}$ show encouraging results regarding the transmission of infrared (IR) light close to $3 \mu \mathrm{m}$. The main purpose of this study was to investigate some of the fibers known to efficiently transmit Er:YAG laser energy at a wavelength of $2.94 \mu \mathrm{m}$. For this study, we chose four different fibers to couple an Er:YAG laser. Transmission efficiency, resistance to high-power laser energy, and bending flexibility are the key criteria to consider when selecting a fiber for endoscopic laserosteotomy, especially for deep bone ablation.

\section{Materials and Methods}

\subsection{Laser Source and Optical Fibers}

The laser source used in this study was a microsecond high-power Er:YAG laser (Syneron Litetouch) with wavelength $\lambda=2.94 \mu \mathrm{m}$. The energy per pulse of the laser could be set within the range 100 to $820 \mathrm{~mJ}$, with a repetition rate range of 1 to $50 \mathrm{~Hz}$ and a pulse duration range of 100 to $400 \mu \mathrm{s}$. We studied the performance of four different fibers in terms of (1) the transmission efficiency and its stability over time; (2) the input tip temperature; and (3) the bending stability of the fibers over time. The fibers chosen for our study are known to have high transmission in the mid-IR wavelength range. To compare performance, we used fibers with similar core sizes and a 1-m length. The glass-based fibers were made of germanium oxide $\left(\mathrm{GeO}_{2}\right)$, sapphire $\left(\mathrm{Al}_{2} \mathrm{O}_{3}\right)$, zirconium fluoride glass $\left(\mathrm{ZrF}_{4}\right)$, and a hollow-core silica waveguide, respectively. Table 1 displays the main properties of the fibers used.

"Germanium" fibers consist of a germanium oxide $\left(\mathrm{GeO}_{2}\right)$ glass core, a glass cladding, a polyamide coating, and a thermoplastic coating in the external layer. "Sapphire" $\left(\mathrm{Al}_{2} \mathrm{O}_{3}\right)$ fibers 
Table 1 Properties of the optical fibers used.

\begin{tabular}{lcccc}
\hline \hline & $\begin{array}{c}\text { Germanium } \\
\left(\mathrm{GeO}_{2}\right)\end{array}$ & $\begin{array}{c}\text { Sapphire } \\
\left(\mathrm{Al}_{2} \mathrm{O}_{3}\right)\end{array}$ & $\begin{array}{c}\text { Fluoride } \\
\left(\mathrm{ZrF}_{4}\right)\end{array}$ & $\begin{array}{c}\text { Hollow-core } \\
\text { silica }\end{array}$ \\
\hline Core size $(\mu \mathrm{m})$ & 450 & 425 & 400 & 500 \\
$\mathrm{NA}$ & 0.25 & 0.12 & 0.2 & 0.025 \\
Attenuation $(\mathrm{dB} / \mathrm{m})$ & 0.5 & 0.25 & 0.015 & 1.5 \\
Min. bending rad $(\mathrm{cm})$ & 4 & 8 & 4.5 & 15 \\
Manufacturer & $\begin{array}{c}\text { Infrared Fiber } \\
\text { Systems }\end{array}$ & $\begin{array}{c}\text { Laser } \\
\text { Components }\end{array}$ & $\begin{array}{c}\text { Le Verre } \\
\text { Fluoré }\end{array}$ & $\begin{array}{c}\text { Laser } \\
\text { Components }\end{array}$ \\
\hline \hline
\end{tabular}

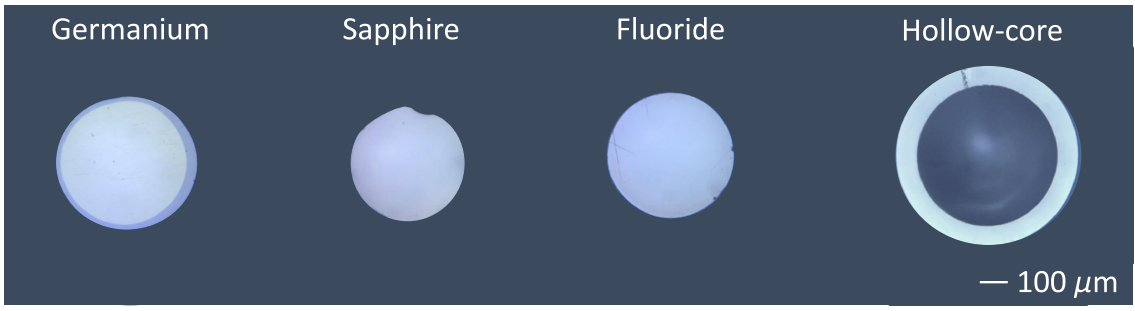

Fig. 1 Microscopic images of the fibers' polished surfaces.

have neither cladding nor sheathing. "Fluoride" fibers are made of a zirconium fluoride $\left(\mathrm{ZrF}_{4}\right)$ glass core and have a double cladding. The second cladding is made of low index resin. It has an external polyacrylate sheathing and germanate end caps. End-capping is a technology in which a different material is spliced into the fiber tip to reduce the power density at the end tips, thereby decreasing the heat as well. The inner wall of "hollow-core" fiber is made of a silver iodide (AgI) reflector, covered by a silica tube and an external acrylate buffer. All of the fibers are commercially available, except for the fluoride one. Currently, fluoride fibers are in a testing phase. Figure 1 shows the end tips of the fibers when they are polished. The end tips were observed with a fiber inspection scope (Thorlabs, FS200) and images were taken using a mobile phone camera.

\subsection{Coupling the Laser into the Fibers}

Figure 2 shows a diagram of the setup used for our study. The setup diagram is divided into four parts, representing the sequence of steps performed during our experiments. The coupling process corresponds to steps (1a) and (1b). Two coupling mirrors $\mathbf{M}_{1}$ and $\mathbf{M}_{2}$ (1a) were used to optimize the coupling process. In $(1 \mathrm{~b})$, a calcium fluoride $\left(\mathrm{CaF}_{2}\right)$ focusing lens with 25.4-mm focal length was mounted to an $X Y$ translating lens mount. The optical fiber was inserted in a fiber chuck for bare fibers (Newport, FPH-DJ), placed on a $Z$ translating mount and an $X Y Z \theta_{x} \theta_{y}$ fiber optic positioner (Newport, FP-2A). With a laser $\mathbf{M}^{2}$ of 22 , the lens provided a beam size of $292 \mu \mathrm{m}$ at the focal plane. The divergence of the beam was $\theta=0.14 \mathrm{rad}$, which fulfilled the requirements of the germanium and the fluoride fibers; it was a little high for the sapphire fiber whose numerical aperture (NA) was 0.12 , and too high for the hollow-core fiber (NA $=0.025$ ). In previous experiment, ${ }^{21}$ a beam size of $328 \mu \mathrm{m}$ and a suitable beam divergence of $0.12 \mathrm{rad}$ for the sapphire fiber was used; however, the fiber could not withstand more than $300 \mathrm{~mJ}$. According to the following equation for calculating the beam size $D_{0}$,

$$
D_{0}=\frac{2 \lambda M^{2}}{\pi \theta}
$$




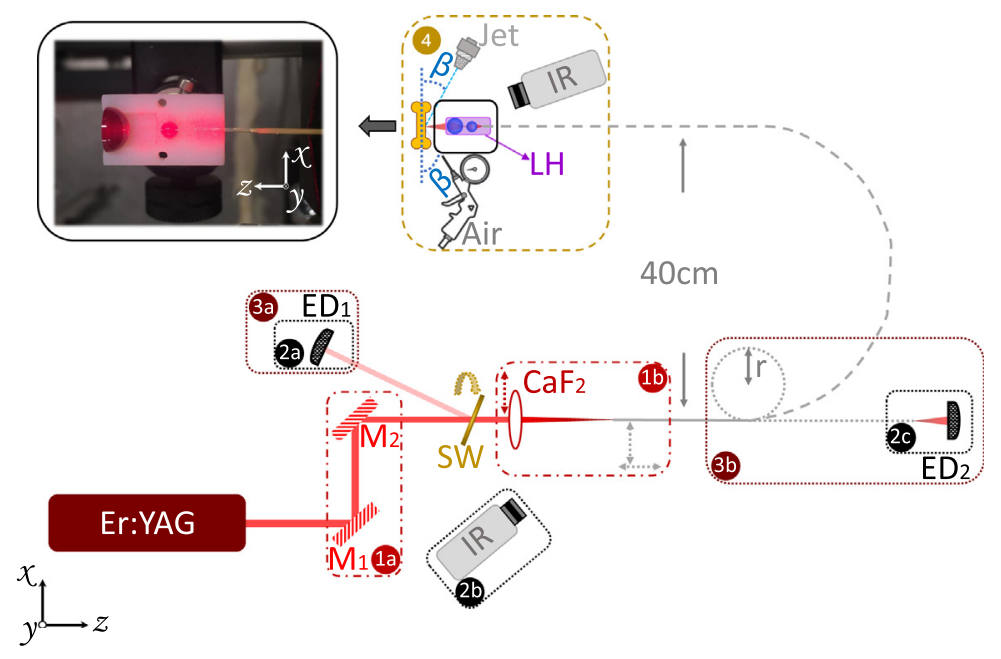

Fig. 2 Schematic of the setup used for studying the performance of several glass fibers for transmitting Er:YAG laser light. The laser was coupled to an optical fiber by means of two coupling mirrors (1a) and a coupling $\mathrm{CaF}_{2}$ lens (1b). A portion of the initial energy was measured with an energy meter $E_{1}(2 a)$. The transmitted energy was measured with an energy meter $E_{2}(2 c)$. While the reflected and the transmitted energy were being measured, an IR camera recorded the temperature of the fiber input face (2b). The bending stability of the fibers was tested by bending the fibers to different radii $r(3 b)$, the transmission efficiency was also determined by the reflected energy measured with $E D_{1}(3 a)$, and the transmitted energy measured with $\mathrm{ED}_{2}(3 \mathrm{~b})$. The ablation performance of the laser through one of the fibers was studied (4). An IR camera was used as a temperature feedback system to automatically control a water jet irrigation system. The water jet (diameter $50 \mu \mathrm{m})$ was placed at an angle $(\beta=30 \mathrm{deg})$ in the upper right quadrant of the $X Z$-plane. A pressurized air gun was placed at an angle $(\beta=30 \mathrm{deg})$ in the lower right quadrant of the $X Z$-plane. The photograph next to (4) presents the final lenses and holder design used for ablation.

to satisfy $\theta \leq \mathrm{NA}$ for the hollow-core fiber with the current laser, the beam size at the tip of the fiber had to be $\geq 1.6 \mathrm{~mm}$, exceeding the size of its $500-\mu \mathrm{m}$ hollow core. These facts regarding the sapphire and the hollow-core fibers were taken into account in the subsequent experiments.

\subsection{Transmission Efficiency over Energy}

In this section, we describe the transmission efficiency over energy for each fiber for 100 pulses at 5 and $10 \mathrm{~Hz}$. To determine the ratio of transmitted energy over input energy, we used two energy meters $\mathrm{ED}_{1}$ and $\mathrm{ED}_{2}$, depicted in Fig. 2. To determine the input energy, we used a sapphire window (SW) to reflect a small percentage of the beam into the first energy detector $\mathrm{ED}_{1}$, step (2a). The reflected percentage from the $\mathrm{SW}$ was about $0.5 \%$. The transmitted energy was measured directly with the second energy detector $\mathrm{ED}_{2}$, step (2c).

\subsection{Transmission Stability over Time and Temperature Monitoring}

At the maximum level of energy transmitted by each fiber at 5 and $10 \mathrm{~Hz}$, we studied the stability of the transmission over $5 \mathrm{~min}$. While measuring the reflected and transmitted energies [Fig. 2, steps (2a) and (2c), respectively], we monitored the temperature at the fiber input tip, using an IR camera, FLIR A655sc at $50 \mathrm{fps}$, step (2b).

\subsection{Bending Stability}

Fiber flexibility and transmission efficiency were studied by bending the fiber to different radii $r$, as depicted in Fig. 2, until reaching the minimum bending radius reported by the manufacturer 
for each fiber (Table 1). The two detectors, $\mathrm{ED}_{1}$ in step (3a) and $\mathrm{ED}_{2}$ in step (3b), were used to determine the transmission efficiency of the bent fiber over time. The measurement was performed for $5 \mathrm{~min}$ at each bending radius.

\subsection{Miniaturized Focusing System for Appropriate Ablation through Fiber}

The ablation of bone in free space, using our Er:YAG laser, had already been optimized for a beam size of $526 \mu \mathrm{m} .{ }^{11}$ We designed a lens system capable of focusing the beam in a manner similar to that of the previous study's free-space setup. ${ }^{11}$ Since beam propagation differs depending on the type of fiber it passes through (due to different output NA), the lens was designed for the fiber that we considered most suitable for endoscopic laser surgeries, based on the experimental findings. Figure 2, step (4) shows a picture of the lens design used for the ablation experiments. The design is described in more detail in Sec. 3.4.

\subsection{Bone Ablation Performance in Minimally Invasive Settings}

Figure 2, step (4) depicts the bone ablation process. The samples used were sheep tibia cortical bone from the Musculoskeletal Research Unit at the University of Zürich. The cadaveric sheep bones were obtained from an animal euthanized in the context of an experiment unrelated to the study reported herein. The cadaveric samples were used within $84 \mathrm{~h}$ after the animal was euthanized, and kept moist at all times before ablation. Three ablation lines, 10-mm long, were each created over a 4-min period. The irrigation system was composed of a Maximator pump ref No. MP030066 and a nozzle from Synova Laser MicroJet Technology. The nozzle produced a water jet with a diameter of $50 \mu \mathrm{m}$. For the experiments, the pump ran at $30 \mathrm{bar}$ pressure. Pressurized air of 2 bar was used to blow off the debris and the remaining water from the ablated area. The IR camera described in Sec. 2.4 was used to monitor the superficial temperature of the bone. Immediately after ablation, the samples were preserved in $4 \%$ formalin. One week later, the samples were taken out for a short time to measure the depth and width of the ablated areas. This measurement was performed using an optical coherence tomography (OCT) system available in our laboratory. The OCT system was composed of an Axsun swept laser with a 1064-nm central wavelength and a 100-nm bandwidth. B-scans to analyze the images were taken with a 14.5-mm field of view and 3.51-mm imaging depth.

The ablation setup used in our study, comprising an automated temperature feedback mechanism (IR camera) for the irrigation system (thin water jet) and pressurized air, was described in detail in our previous study. ${ }^{11}$

\subsection{Histology}

Sheep cortical bone samples were used to create cuts using the Er:YAG laser through an optical fiber. The non-decalcified bone samples were fixed in $4 \%$ buffered formalin, then dehydrated in an ascending series of ethanol, after which, the samples were defatted in xylene and immersed in methylmethacrylate under vacuum; final polymerization was reached after complete infiltration. Thick sections (200 to $700 \mu \mathrm{m}$ ) were cut using a diamond saw (Exact ${ }^{\circledR} 310$ saw), mounted on Acropal slides and surface stained with toluidine blue. The sections were then imaged using a macroscope (Z6 APO A, Leica Microsystems) with a DFC450 Digital Camera (Leica Microsystems). For higher magnifications, we used a DMR microscope system (Leica Microsystems) with a DC320 digital camera (Leica Microsystems).

\section{Results and Discussion}

\subsection{Transmission Efficiency over Energy}

Figure 3 shows the transmission efficiency over the input energy of the laser, obtained for each fiber at 5 and $10 \mathrm{~Hz}$. Each data point on the graph is the average over 100 pulses with the corresponding standard deviation. 


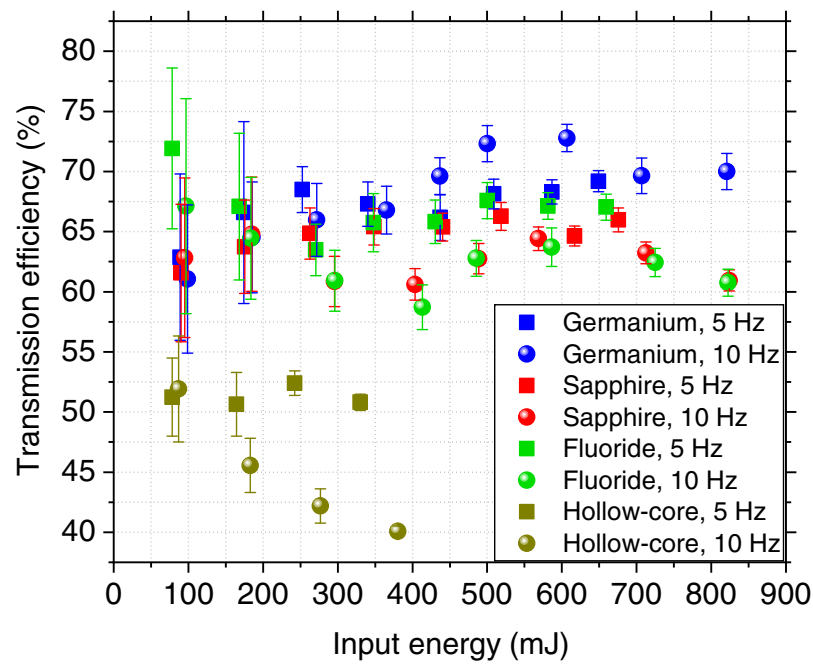

Fig. 3 Transmission efficiency of germanium oxide, sapphire, zirconium fluoride, and hollow-core silica fibers at 5 and $10 \mathrm{~Hz}$ at different input energies.

During our experiments, the transmission efficiency obtained for the sapphire fiber was $64 \% \pm 2 \%$; however, other researchers have shown that the sapphire fiber can reach up to $90 \%$ transmission efficiency. ${ }^{18}$ The relatively low performance in our study was most likely caused by a beam divergence that did not completely fulfill the NA requirements of the sapphire fiber. As seen in Fig. 3, the fluoride fiber achieves higher transmission at low energies and then tapers off; its transmission efficiency was $65 \% \pm 3 \%$. The hollow-core fiber tip burned at energies higher than $400 \mathrm{~mJ}$, resulting in the need to unmount and polish the fiber. The maximum input energy for the hollow-core fiber (without burning) was 330 and $380 \mathrm{~mJ}$ at 5 and $10 \mathrm{~Hz}$, respectively. The lowest transmission was observed in the hollow-core fiber; at $5 \mathrm{~Hz}$, the transmission efficiency was only $51 \% \pm 1 \%$, while at $10 \mathrm{~Hz}$, the transmission was $45 \% \pm 5 \%$, decreasing over the energy. NA matching issues account in part for the low performance of the hollow-core fiber. Of the fibers used, the germanium fiber showed the highest transmission efficiency at $68 \% \pm 3 \%$, similar to that achieved in other studies. ${ }^{22}$

\subsection{Transmission Stability over Time and Monitoring the Input Tip Temperature}

After examining the behavior of the fibers exposed to different input energies, the transmission stability of the fibers was tested for $300 \mathrm{~s}(5 \mathrm{~min})$. We applied the laser's maximum achievable energy level, about $820 \mathrm{~mJ}$, to the input fiber face. Since the hollow-core fiber could not withstand such high energy levels, we used energy settings of 330 and $380 \mathrm{~mJ}$ at 5 and $10 \mathrm{~Hz}$, respectively, for this fiber.

Figure 4 shows the evolution of the transmission efficiency of each fiber over 5 min at 5 and $10 \mathrm{~Hz}$. The values (black data points) presented in each graph are an average of five individual measurements, the gray areas represent the corresponding standard deviation.

In general, all of the fibers showed stable energy transmission over time. The fluoride fiber showed a decrease in energy transmission in the first $100 \mathrm{~s}$, but stabilized thereafter.

The input face temperature of each fiber was monitored while simultaneously measuring its energy transmission. Figure 5 shows the average temperature of each fiber tip.

At $10 \mathrm{~Hz}$, the temperature of the germanium fiber dropped within the first $100 \mathrm{~s}$; this is in part because the energy of the laser drops a little over time. The drop in temperature probably also reflects a decrease in the fiber material's heat capacity around the average temperature of $70^{\circ} \mathrm{C}$, measured with our thermal camera. At lower temperatures, there was no noticeable change in the material's heat capacity, as observed for both the germanium oxide fiber at $5 \mathrm{~Hz}$ and for the other fibers. The sapphire fiber tip's temperature increased the least among all of the fibers. Additionally, sapphire's physical properties make it highly resistant to scratches and fractures. ${ }^{23}$ 


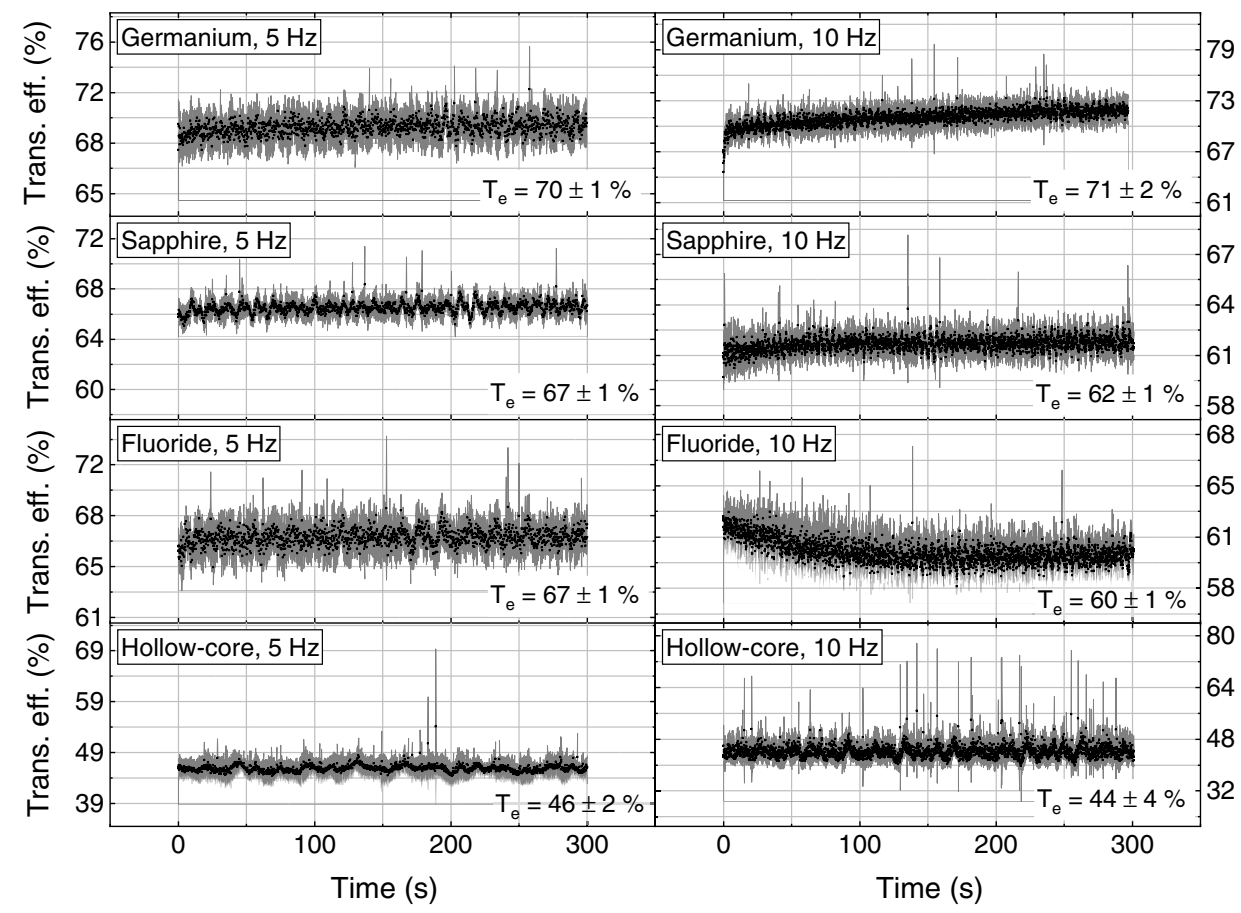

Fig. 4 Transmission efficiency over time of each fiber at 5 and $10 \mathrm{~Hz}$. The input energy was $820 \mathrm{~mJ}$ for all fibers except the hollow-core fiber, which was set at 330 and $380 \mathrm{~mJ}$ at 5 and $10 \mathrm{~Hz}$, respectively.
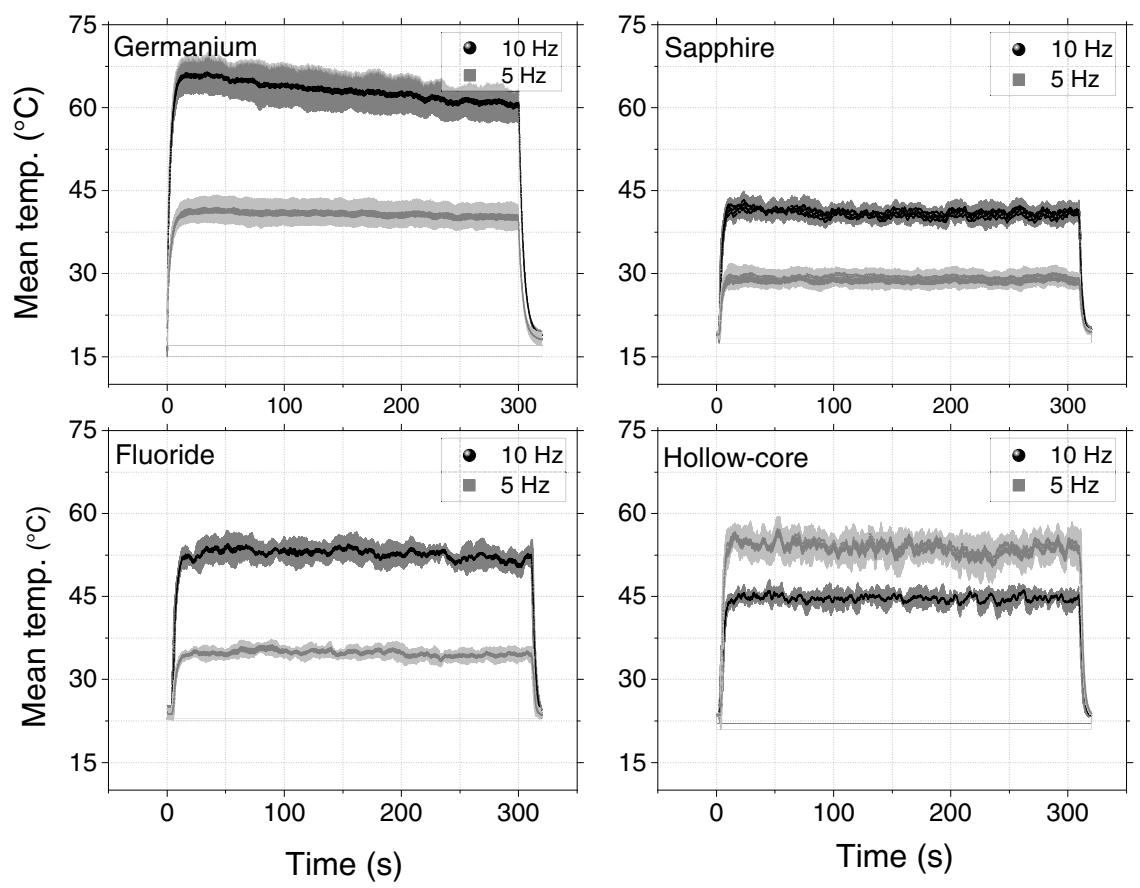

Fig. 5 Evolution of the temperature of each input fiber tip, monitored for $300 \mathrm{~s}$. The input energy of the laser was $820 \mathrm{~mJ}$ for all fibers except the hollow-core fiber, which was set at 330 and $380 \mathrm{~mJ}$ at repetition rates 5 and $10 \mathrm{~Hz}$, respectively. 
Therefore, for endoscopic applications, one may consider using sapphire fibers or splicing them in other fibers. ${ }^{24}$ Doing so may reduce the heat at the endoscope's tip, thereby also reducing the risk of damaging sensitive material and making it easier to manipulate the fiber.

\subsection{Bending Stability}

After the fibers were tested for transmission efficiency, behavior over time, and input tip temperature over time, we bent the fibers from a straight position up to the minimum bending radii reported by the manufacturers.

Figure 6 shows the effect of bending the fibers on transmission efficiency. As in Sec. 3.2, we delivered the maximum possible energy to the fibers. Each individual data point in the graph is the average over $5 \mathrm{~min}$ of five individual measurements.

As seen in Fig. 6, the transmission efficiency of the fibers does not change remarkably when bent to the minimum radius possible, except in the case of the sapphire fiber. At its minimum bending radius, the transmission of the sapphire fiber dropped from $60 \%$ to $53 \%$ at $10 \mathrm{~Hz}$ and from $67 \%$ to $60 \%$ at $5 \mathrm{~Hz}$. The transmitted energy dropped in an unusual way for the bent fluoride fiber: the fiber tip was burnt and had to be polished. The transmission efficiency of the fluoride fiber at a $4.5-\mathrm{cm}$ bending radius, as reported in Fig. 6, corresponds to the response of the fiber after repair.

\subsection{Bone Ablation Performance in Minimally Invasive Settings}

Based on the properties of the fibers (Table 1) and the studies realized in the previous sections, we found that the germanium oxide fiber fulfilled most of the requirements for use in endoscopic surgical applications. The germanium fiber is biocompatible, showed slightly higher transmission efficiency than the other fibers (Figs. 3 and 4), and is the most flexible among the fibers tested; its minimum bending radius is $4 \mathrm{~cm}$. The only disadvantage of the germanium oxide fiber relative to the others is the high tip temperature reached while the laser was being coupled. However, this issue can be solved by choosing coupling and refocusing materials (for lenses and holders) that dissipate the heat efficiently, or by adding a cooling system to the fiber. The fiber tips can be treated, for instance. Previous studies have shown that the germanium fiber can be spliced with sapphire, although doing so reduced its transmission from $68 \%$ to $65 \%$. $^{22}$

In this section, we present a refocusing system designed for the germanium fiber. The refocusing system consisted of two lenses inserted into a holder. This system was used for ablating

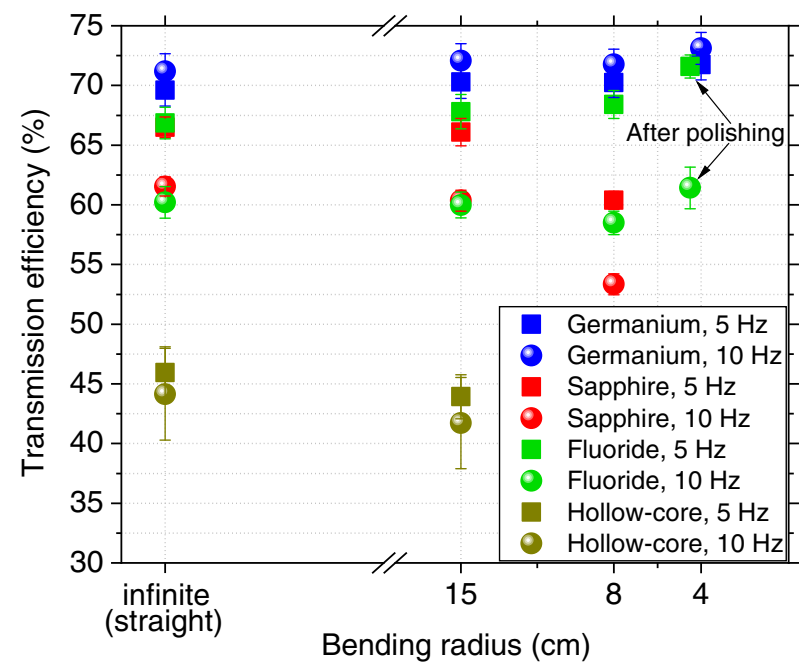

Fig. 6 Transmission efficiency of each fiber at different bending radii. The transmission efficiency of the fluoride fiber at $4.5 \mathrm{~cm}$ radius corresponds to the response of the fiber after polishing its surface. The input energy of the laser was $330 \mathrm{~mJ}$ (at $5 \mathrm{~Hz}$ ) and $380 \mathrm{~mJ}$ (at $10 \mathrm{~Hz}$ ) for the hollowcore fiber, and $820 \mathrm{~mJ}$ for the others. The repetition rate of the laser was 5 and $10 \mathrm{~Hz}$. 
(a)

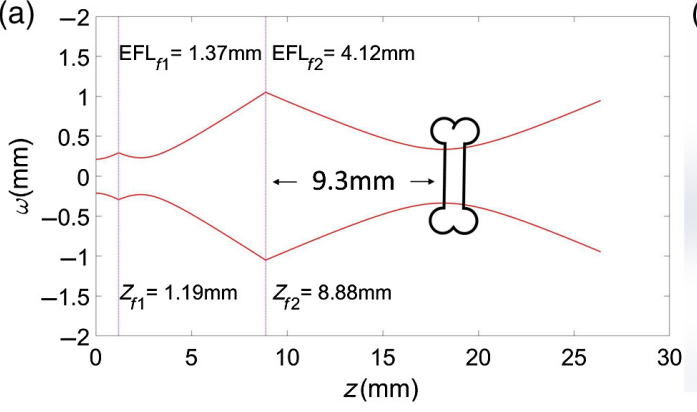

(b)

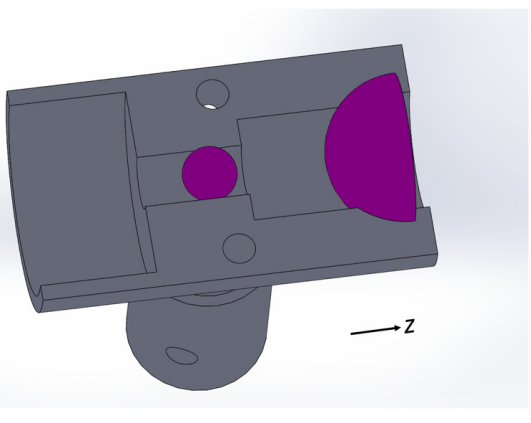

Fig. 7 (a) Schematic of the propagation of the beam after the fiber and the two focusing lenses (represented by the two vertical dashed lines) have been inserted, calculated using MATLAB. (b) Lenses and holder design made in SolidWorks.

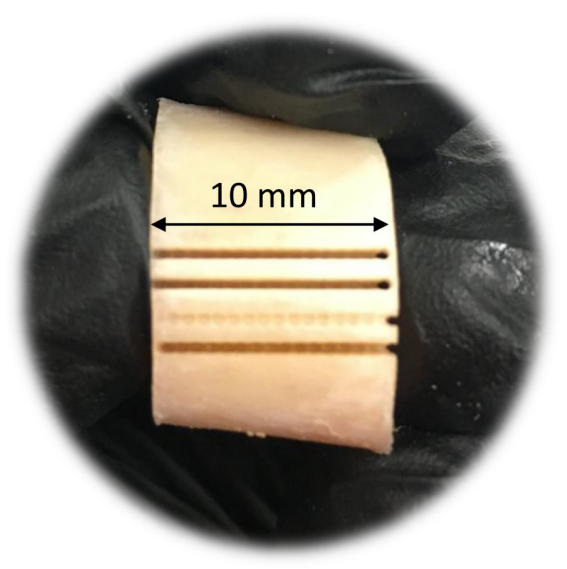

Fig. 8 Photograph of one of the sheep bones ablated with the Er:YAG laser through a germanium oxide fiber. All of the lines have a length of $10 \mathrm{~mm}$. The first two lines and the fourth one were made by scanning the bone 180 times (about $4 \mathrm{~min}$ at a speed of $8 \mathrm{~mm} / \mathrm{s}$ ). The third line was ablated with only one laser scan.

sheep bones. Figure 7 shows a schematic of beam propagation after the fiber and the two focusing lenses have been inserted, such that the beam focuses on the bone surface at almost $10 \mathrm{~mm}$ beyond the last lens. The lenses were ruby-doped sapphire ball and half-ball lenses from Edmund Optics. The corresponding effective focal lengths were $\mathrm{EFL}_{f 1}=1.37 \mathrm{~mm}$ and $\mathrm{EFL}_{f 2}=4.12 \mathrm{~mm}$. The first lens was a ball lens with a diameter of $2.38 \mathrm{~mm}$, while the second lens was a half-ball lens with a diameter of $6.35 \mathrm{~mm}$. This lens system provides a beam diameter of $673 \mu \mathrm{m}$ at the focal plane, located $9.3 \mathrm{~mm}$ away from the last lens.

The lens holder was 3D-printed in VeroWhitePlus, RGD835 material. This material is not biocompatible and, therefore, not recommended for real surgery applications. However, it was a very useful prototype and served to identify the benefits of our system, as well as the issues to be improved.

Figure 8 shows a photograph of a sheep bone ablated with the Er:YAG laser coupled with the germanium oxide fiber. For ablation, the maximum achievable energy of the laser beyond the lenses was $370 \mathrm{~mJ}$ at a $10-\mathrm{Hz}$ repetition rate. The ablation depth achieved through the germanium oxide fiber was $6.82 \pm 0.99 \mathrm{~mm}$, while the width was $0.69 \pm 0.06 \mathrm{~mm}$.

\subsection{Histology Analysis}

As shown in Fig. 9, the laser was able to cut through sheep cortical bone in a straight path through the bone lamellae. The structural features of the cortical bone adjacent to the laser path remained intact, as seen in Fig. 9(c). 


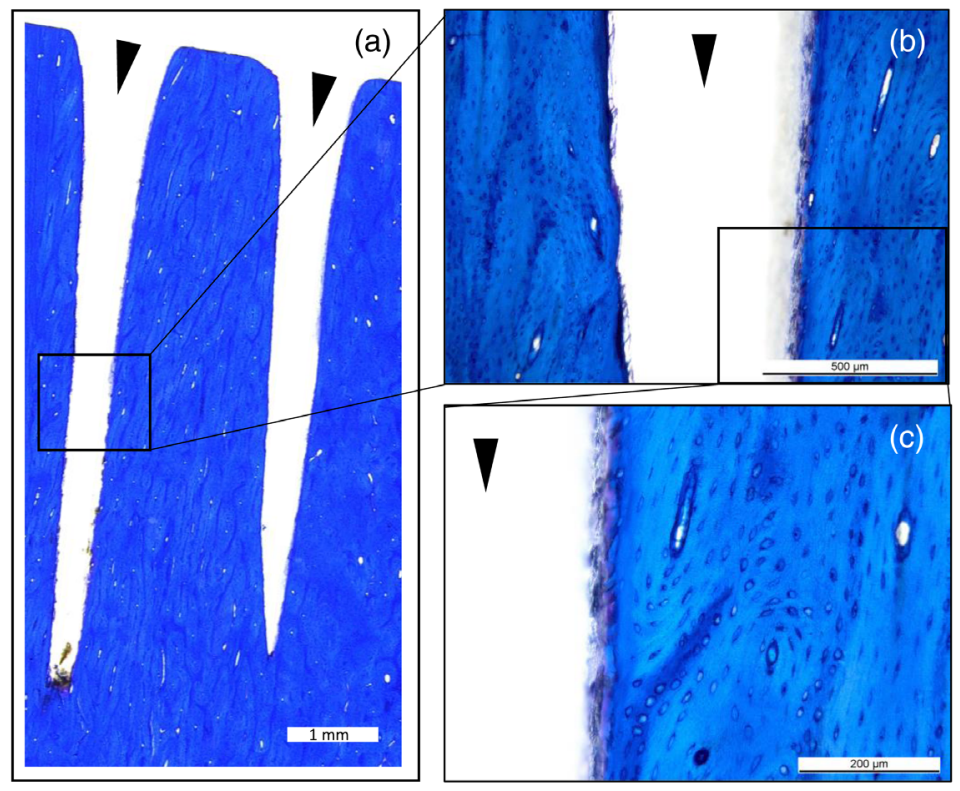

Fig. 9 Sheep cortical bone with two cuts made with the Er:YAG laser through $\mathrm{GeO}_{2}$ fiber, shown in panel (a). Higher magnifications in panel (b) and panel (c) show the bone tissue interface with the laser's path, as well as the tissue structure. The toluidine blue stain highlights the cells within the bone and the lamellar structure, and shows how the laser can cut through these structures in a controlled manner. The black arrows indicate the laser cuts. The scale bars indicate $1 \mathrm{~mm}$, $500 \mu \mathrm{m}$, and $200 \mu \mathrm{m}$ in panels (a), (b), and (c), respectively.

\section{Conclusion}

Four fibers suitable for transmitting high-power laser energy in the mid-IR region of the spectrum were used for coupling an Er:YAG laser at a wavelength of $2.94 \mu \mathrm{m}$. The setup was not optimal for the sapphire and hollow-core fibers, since the divergence of the beam did not fully match the required NA. However, the sapphire fiber remained undamaged at the laser's maximum energy of $820 \mathrm{~mJ}$. The hollow-core fiber could only withstand a maximum energy of $380 \mathrm{~mJ}$, and the germanium fiber had the highest transmission efficiency at $68 \% \pm 3 \%$, as shown in Fig. 3. Transmission efficiency was stable for $5 \mathrm{~min}$ at $820 \mathrm{~mJ}$ for all but the hollow-core fiber, which was stable at $380 \mathrm{~mJ}$. However, the fluoride fiber showed a drop in efficiency after the first $100 \mathrm{~s}$ (Fig. 4). Figure 5 shows the input tip temperature over time. In general, it was stable for all fibers, however, the maximum average temperature of the germanium fiber's tip showed higher temperature around $70^{\circ} \mathrm{C}$ compared to the other fibers. When decreasing the bending radius of the fluoride fiber to $4.5 \mathrm{~cm}$, we observed a drop in the transmitted energy (Fig. 6) and an increase in the temperature of the tip. Examination of the fiber surface through a fiber microscope confirmed the suspicion that the fiber had been damaged. Based on the reported results, and on the results from our previous studies ${ }^{21}$ we consider the germanium oxide fiber to be the most appropriate choice for future endoscopic applications. Still, the temperature issue must be resolved, for instance, by splicing the end tips with sapphire. We designed a lens system capable of focusing the laser beam after the fiber, at nearly $10 \mathrm{~mm}$ beyond the last lens, with a beam diameter of $673 \mu \mathrm{m}$. At the maximum achievable output energy of $370 \mathrm{~mJ}$ at $10 \mathrm{~Hz}$, we obtained an ablation depth of $6.82 \pm 0.99 \mathrm{~mm}$ in sheep bone. At present, the small working space of the fiber setup limits our ability to align the water jet and pressurized air with the laser beam. Therefore, the irrigation and cleaning design must be adapted to small working distances of less than $10 \mathrm{~mm}$. Histology image analysis showed no structural damage on the surroundings of the cut made with the Er:YAG laser through the $\mathrm{GeO}_{2}$ fiber (Fig. 9). The fiber system presented in this study has the potential to be implemented in endoscopic laserosteotomy systems. 


\section{Disclosures}

The authors have no conflicts of interest to declare in this paper.

\section{Acknowledgments}

The authors gratefully acknowledge funding from the Werner Siemens Foundation through the Minimally Invasive Robot Assisted Computer-guided LaserosteotomE (MIRACLE) project. The authors gratefully acknowledge Mr. Alex Tchap from Infrared Fiber Systems for providing us the $\mathrm{GeO}_{2}$ fibers to perform our experiments and Mr. Samuel Poulain, Dr. Solenn Cozic, and Ms. Laurine Bodin from Le Verre Fluoré for providing us the double clad fluoride fiber.

\section{Code, Data, and Materials Availability}

The data generated and analyzed in this study are included in this paper.

\section{References}

1. S. R. Visuri, J. T. Walsh, and H. A. Wigdor, "Erbium laser ablation of dental hard tissue: effect of water cooling," Lasers Surg. Med. 18(3), 294-300 (1996).

2. K. Stock, R. Hibst, and U. Keller, "Comparison of Er:YAG and Er:YSGG laser ablation of dental hard tissues," Proc. SPIE 3192, 88-96 (1997).

3. M. Werner et al., " $\mathrm{Co}_{2}$ laser free-form processing of hard tissue," in Eur. Conf. Biomed. Opt., Paper 6632_1, Optical Society of America (2007).

4. L. Kuščer and J. Diaci, "Measurements of erbium laser-ablation efficiency in hard dental tissues under different water cooling conditions," J. Biomed. Opt. 18(10), 108002 (2013).

5. K. Stock, H. Wurm, and F. Hausladen, "Primary investigations on the potential of a novel diode pumped Er:YAG laser system for middle ear surgery," Proc. SPIE 9689, 96892K (2016).

6. N. Jowett et al., "Bone ablation without thermal or acoustic mechanical injury via a novel picosecond infrared laser (PIRL)," Otolaryngology 150(3), 385-393 (2014).

7. K.-W. Baek et al., "A comparative investigation of bone surface after cutting with mechanical tools and Er:YAG laser," Lasers Surg. Med. 47(5), 426-432 (2015).

8. M. Papadaki et al., "Vertical ramus osteotomy with Er:YAG laser: a feasibility study," Int. J. Oral Maxillofacial Surg. 36(12), 1193-1197 (2007).

9. B. Fink et al., "Holmium:YAG laser-induced aseptic bone necroses of the femoral condyle," Arthroscopy 12(2), 217-223 (1996).

10. S. Stübinger et al., "Er:YAG laser osteotomy: preliminary clinical and histological results of a new technique for contact-free bone surgery," Eur. Surg. Res. 42(3), 150-156 (2009).

11. L. M. B. Bernal et al., "Optimizing deep bone ablation by means of a microsecond Er:YAG laser and a novel water microjet irrigation system," Biomed. Opt. Express 11(12), 7253-7272 (2020).

12. K.-W. Baek et al., "Clinical applicability of robot-guided contact-free laser osteotomy in cranio-maxillo-facial surgery: in-vitro simulation and in-vivo surgery in minipig mandibles," Br. J. Oral Maxillofacial Surg. 53(10), 976-981 (2015).

13. W. Deibel et al., "A compact, efficient, and lightweight laser head for Carlo ${ }^{\circledR}$ : integration, performance, and benefits," Proc. SPIE 9579, 957905 (2015).

14. T. Borus and T. Thornhill, "Unicompartmental knee arthroplasty," J. Am. Acad. Orthopaedic Surg. 16(1), 9-18 (2008).

15. G. Berci and K. A. Forde, "History of endoscopy," Surg. Endosc. 14(1), 5 (2000).

16. G. Iddan et al., "Wireless capsule endoscopy," Nature 405(6785), 417 (2000).

17. N. J. Scott et al., "Mid-IR germanium oxide fibers for contact erbium laser tissue ablation in endoscopic surgery," IEEE J. Sel. Top. Quantum Electron. 13(6), 1709-1714 (2007).

18. J. Qiu et al., "Comparison of fluoride and sapphire optical fibers for Er:YAG laser lithotripsy," J. Biophotonics 3(5-6), 277-283 (2010). 
19. M. Sofer et al., "Holmium:YAG laser lithotripsy for upper urinary tract calculi in 598 patients," J. Urol. 167(1), 31-34 (2002).

20. R. L. Blackmon, P. B. Irby, and N. M. Fried, "Holmium:YAG $(\lambda=2,120 \mathrm{~nm})$ versus thulium fiber ( $\lambda=1,908 \mathrm{~nm}$ ) laser lithotripsy," Lasers Surg. Med. 42(3), 232-236 (2010).

21. L. M. B. Bernal et al., "Measurements of coupling efficiency of high power Er:YAG laser in different types of optical fibers," Proc. SPIE 11233, 112331H (2020).

22. T. J. Polletto et al., "Comparison of germanium oxide fibers with silica and sapphire fiber tips for transmission of erbium:YAG laser radiation," Lasers Surg. Med. 38(8), 787-791 (2006).

23. E. R. Dobrovinskaya, L. A. Lytvynov, and V. Pishchik, Sapphire: Material, Manufacturing, Applications, Springer Science \& Business Media, Germany (2009).

24. Y. O. Aydin et al., "Endcapping of high-power $3 \mu \mathrm{m}$ fiber lasers," Opt. Express 27(15), 20659-20669 (2019).

Lina M. Beltrán Bernal received her BSc degree in physics from the Universidad Nacional de Colombia (UNAL), Bogotá D.C., Colombia, in 2013 and her MSc (Hons) degree in advanced optical technologies from the Friedrich-Alexander-Universität Erlangen-Nürnberg (FAU), Erlangen, Germany, in 2016, and her $\mathrm{PhD}$ in biomedical engineering from the Universität Basel, Basel, Switzerland, in 2021. Her main research interests include laser technology, design of optical systems for the development smart devices, and smart laser surgery.

Ferda Canbaz is a postdoctoral researcher in the Department of Biomedical Engineering at University of Basel. She received her BS degree in engineering physics from Istanbul Technical University, Istanbul, Turkey, in 2011. She received her MS degree in optoelectronics and photonics engineering in 2013 and her PhD in physics in 2018 from Koç University, Istanbul, Turkey. Her research interests include mid-infrared solid-state/fiber lasers, ultrafast optics, photoacoustic diagnosis, and smart laser surgery.

Salim E. Darwiche received his bachelor's and master's degree in bioengineering from the University of Pennsylvania in 2006 and 2008, respectively. He then completed his PhD in biotechnology and bioengineering from the École polytechnique Fédérale de Lausanne in 2013. Since 2014, he has worked as a study director and senior scientist at the MSRU. He is also the scientific coordinator of the Center for Applied Biotechnology and Molecular Medicine (CABMM) and as such, acts as a liaison to facilitate collaborations within the consortium of CABMM experts. He has significant experience in designing, implementing, and managing in-vivo studies in compliance with GLP. He has directed and managed many multiyear projects with industrial and academic project partners. He is FELASA certified for directing animal experimentation and has extensive expertise in musculoskeletal tissue (bone, cartilage, and tendon) and skin repair and regeneration.

Katja M. R. Nuss received her degree in veterinary medicine from the University of Münich, Germany, in 1984, she finished her postgraduate studies from the University of Münich in anesthesiology in 1987, intensive care in 1988, and surgery in 1993. She pursued specializations in surgery (1990) and anesthesiology (2000). She became senior staff at the University of Zürich, Vetsuisse Faculty, Musculoskeletal Research Unit (MSRU) from 2004 to 2019 and became a member of the Leadership Consortium of the MSRU since 2020. She has authored over 71 publications in national and international journals.

Niklaus F. Friederich received his MD from the University of Berne, Switzerland in 1981, and finished post-graduate studies in general surgery, anesthesiology, bone radiology and orthopaedic surgery in Switzerland and USA. He is a board certified orthopaedic surgeon (1991) and adjunct professor of orthopaedic surgery of the University of Basel (2003). He was elected Head of the Dept of Orthopaedic Surgery Bruderholz (1998 to 2013), was a co-founder of Orthoklinik Dornach (2013 to 2015) and joined the Department of Orthopaedics \& Traumatology University of Basel (2015-ongoing). He became a medical advisor to the MIRACLE project at the Department of Biomedical Engineering, University of Basel in 2015. In 2019, he was elected visiting professor at the Sunnybrook Health Sciences Center 
in Toronto, Ontario, Canada. Citations: 4819 (since 2016: 1952); h-index 37 (since 2016: 24); i10-index 90 (since 2016: 51).

Philippe C. Cattin received his BSc degree from the University of Applied Science in Brugg/ Windisch, Windisch, Switzerland, in 1991, his MSc degree in computer science, and his $\mathrm{PhD}$ in robotics from ETH Zürich, Zürich, Switzerland, in 1995 and 2003, respectively. From 2003 to 2007, he was a postdoctoral fellow with the Computer Vision Laboratory, ETH Zurich. In 2007, he became an assistant professor with the University of Basel, Allschwil, Switzerland, and was promoted to an associate professor in 2015 and to full professor in 2019 . He is the founder of the Center for Medical Image Analysis and Navigation (CIAN), Medical Faculty, University of Basel, where he is the founding head and still heading the Department of Biomedical Engineering. He joined Brigham and Women's Hospital, Boston, MA, USA, in 2017, as a research fellow. He is also the founder of multiple spin-off companies and licensed his patents and software to medical device companies. His research interests include medical image analysis, image-guided therapy, robotics-guided laser osteotomy, and virtual reality. As a principal investigator, he has finished many projects in these areas and published over 250 papers, patents, and book chapters.

Azhar Zam received his BSc degree in medical physics from the University of Indonesia, Depok, Indonesia, in 2004, his MSc degree in biomedical engineering from the University of Lübeck, Lübeck, Germany, in 2007, and his $\mathrm{PhD}$ in advanced optical technologies from the Friedrich-Alexander University Erlangen-Nuremberg, Germany, with the focus on optical feedback for tissue-specific laser surgery, in 2011. He held various research positions at the University of Waterloo, Canada; the National University of Ireland Galway, Ireland; the University of California at Davis, USA; and Ryerson University, Toronto, Canada. He joined the Department of Biomedical Engineering, University of Basel, Switzerland, in 2016, as an assistant professor, where he founded and leads the Biomedical Laser and Optics Group (BLOG). He has authored over 80 peer-reviewed articles, book chapters, books, and patents. His main research interests focus on the development of smart devices for medical therapy, diagnostics and monitoring using novel optical technologies, including smart laser surgery, optical coherence tomography (OCT), photoacoustics, biomedical spectroscopy, AI-aided optical diagnostics, and imaging, optical-based smart wearable sensors, and miniaturized systems. 